\title{
Frailty, inequality and resilience
}

\author{
Authors: Matthew Hale, ${ }^{A}$ Sarwat Shah ${ }^{B}$ and Andrew Clegg $^{C}$
}

Frailty is a common condition in later life in which minor stressors may result in major changes in health. While the biological mechanisms of frailty are increasingly understood, relationships with the wider determinants of health, health inequalities and the concept of resilience are less well-established and the role of the clinician in their modification is less well understood.

The wider determinants are the modifiable conditions in which people are born, grow, work and live, and the wider set of systems shaping the conditions of daily life. They interact across the life course, driving a well-recognised social gradient in health. The wider determinants are closely linked to the concept of resilience, which is the process of effectively negotiating, adapting to or managing significant sources of stress or trauma. Better recognition of the relationship between frailty, the wider determinants, inequalities and resilience can enable a framework around which policy responses may be developed to build resilience in people living with frailty at an individual and community level as well as enabling clinicians to better identify how they may support their patients.

KEYWORDS: Frailty, inequality, resilience, wider determinants

\section{Introduction}

Frailty is a condition characterised by loss of biological reserves, failure of homeostatic mechanisms and vulnerability to a range of adverse outcomes. ${ }^{1}$ In frailty, a minor stressor, such as an infection or new medication, may dramatically impact an older person's physical, functional or mental wellbeing. The prevalence of frailty increases with age, affecting $4 \%$ of the UK population aged between 65 and 69 years and $26 \%$ of the UK population aged 85 years and over. ${ }^{2}$ At present, 1.8 million people in the UK are estimated to be living with frailty and this is projected to grow over time as both the population size and life expectancy increase. ${ }^{3}$

The best-established models of frailty are the phenotype model, which identifies frailty if three or more physical characteristics (unintentional weight loss, exhaustion, weakness, slow walking speed and low physical activity) ${ }^{4}$ are present in an individual, and the cumulative deficit model, where frailty is identified on the

Authors: ANIHR academic clinical fellow and specialty registrar in geriatrics, Bradford Teaching Hospitals NHS Foundation Trust, Bradford, UK; ${ }^{B}$ research fellow, University of York, York, UK; ${ }^{C}$ clinical senior lecturer and honorary consultant geriatrician, Bradford Teaching Hospitals NHS Foundation Trust, Bradford, UK basis of the accumulation of a range of deficits (signs, symptoms, disease, disabilities and impairments). ${ }^{5}$ People living with frailty experience greater disability, hospitalisation, care home admission and mortality than patients who are not identified as frail. ${ }^{2,5}$ Importantly, there is evidence that frailty has greater reversibility than disability and that important outcomes for older people living with frailty, including falls, nursing home admission and hospitalisation, may be improved through the provision of interventions targeted across the frailty spectrum. ${ }^{6-10}$

\section{Frailty, the wider determinants of health and inequality}

There is increasing understanding of the mechanisms underlying ageing and the development of frailty. ${ }^{2,11}$ It is recognised that these mechanisms are driven by complex interactions between genes and the environment, leading to cumulative molecular and cellular damage across a range of physiological systems. However, the main focus of frailty research has historically been on biological mechanisms and pathways, rather than on the contribution of environmental conditions to the development and progression of frailty.

These environmental conditions are best understood as the wider (or social) determinants of health. These are the conditions in which people are born, grow, work and live, and the wider set of systems shaping the conditions of daily life such as economic and social policies, development agendas and societal norms. ${ }^{12}$ The wider determinants are considered to be the main driver of health inequalities - the unfair and avoidable differences in health among different population groups. ${ }^{13}$ They interact across the life course, driving a well-recognised social gradient in health whereby lower socioeconomic position is associated with worse health outcomes, including mortality ${ }^{14}$ and earlier onset of disability in later life. ${ }^{15}$ Although frailty in older adults is independently associated with individual and neighbourhood socioeconomic factors, ${ }^{16}$ the overall contribution of socioeconomic position to the development and progression of frailty is currently incompletely understood.

More recently, the role of the wider determinants has been incorporated into the 'frailty fulcrum' model, supported by NHS England. ${ }^{17}$ This is an important step because it is likely that the potential contribution of the wider determinants to health and health inequalities in older age are frequently underestimated, particularly by clinicians. However this model does not fully capture how these factors potentially interact, and a schematic representation of the interplay between the wider determinants and other components of frailty is summarised in Fig 1. 


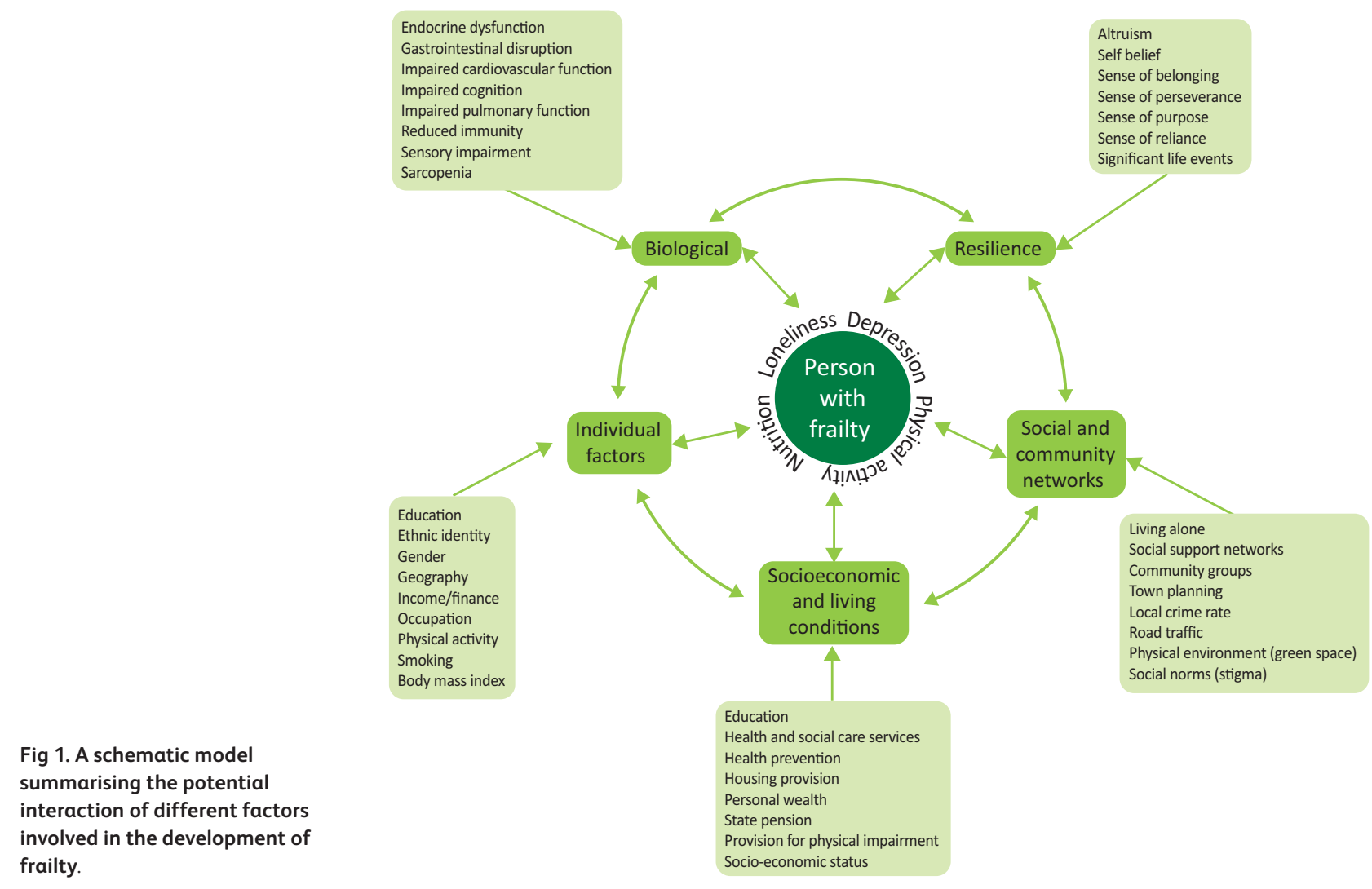

Greater understanding of the wider determinants is important because evidence-based interventions targeted at older people living with frailty, such as comprehensive geriatric assessment, frequently incorporate social context. However, it is presently unclear regarding which of the wider determinants might be the key contributory factors in frailty and which are potentially modifiable through targeted intervention. Fig 2 presents a framework for separating the wider determinants into components which helps inform how interventions may be considered. ${ }^{18,19}$

\section{Individual factors}

Evidence indicates smoking is independently associated with the development and progression of frailty. ${ }^{20}$ Smoking is potentially modifiable, so a continued focus on smoking cessation in older age is appropriate. Low physical activity, sedentary behaviour, being underweight and obesity are also associated with increased risk of development and progression of frailty, ${ }^{21-23}$ and there is evidence that interventions to increase physical activity may improve outcomes for older people with frailty. ${ }^{24}$

\section{Role of the individual clinician}

> Personalised care planning and shared decision making.

> Promoting a healthy lifestyle; including smoking cessation and increased physical activity. This may involve informing individuals of available community groups and involving other specialists such as dieticians, occupational therapists and physiotherapists, as well as enhancing awareness of available home and group based exercise programmes.

\section{Social and community networks}

Increasing frailty has been associated with living alone, ${ }^{25}$ having fewer relatives or neighbours, reduced social contact and the absence of participation in community, religious or altruistic activities. ${ }^{26}$ Despite the importance of these social networks, contact with family and friends has been shown to be lower among persons aged 65 years and older compared to those aged less than 65 years. ${ }^{27}$

Neighbourhood characteristics also contribute significantly to social isolation. Town planning, for example, may increase social isolation by requiring older people to cross busy roads. The risk of having a fatal collision increases with age. ${ }^{28}$ This results in reduced travel patterns, with a fear of leaving the home. ${ }^{29}$ Similarly, a high local rate of crime is associated with increased fear at home and older people leaving their homes less frequently. ${ }^{30}$ These factors not only worsen social isolation but also reduce the opportunity for physical activity. ${ }^{25,26}$ This is in contrast with the presence of nearby green space for older people, which reduces rates of depression and increases activity outside the home. ${ }^{29}$

These wider social factors are often challenging to modify and the effect of interventions targeting them is challenging to investigate. However, benefits have been reported from interventions such as promoting group activities and the use of existing public resources (such as libraries and volunteering groups), one-on-one activities such as befriending and the teaching of IT skills to enable communication using internet-based activities. ${ }^{27}$

\section{Role of the individual clinician}

> Education of patients regarding available community and third sector organisations, activities and befriending schemes. 


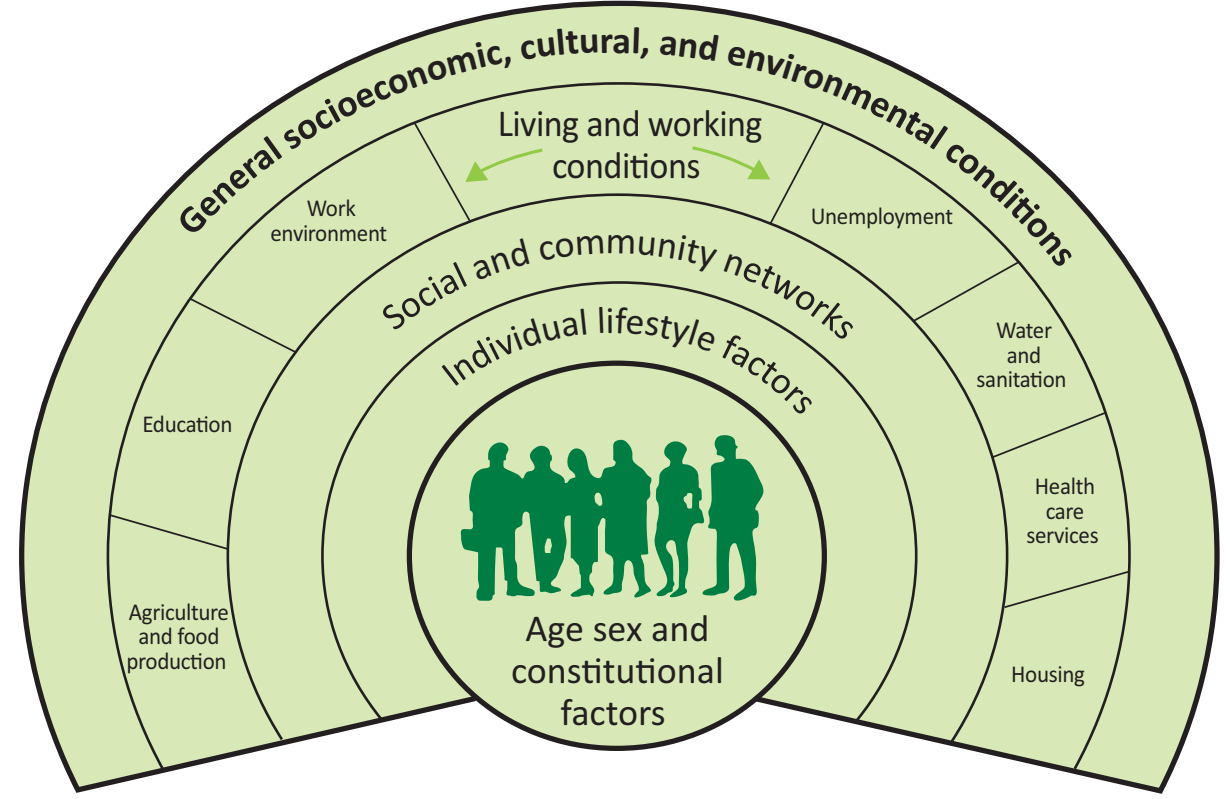

Fig 2. The main determinants of health. Figure adapted with permission from Dahlgren G, Whitehead M. Policies and strategies to promote social equity in health. Stockholm: Institute for Futures Studies, 1991:11.

\section{Socioeconomic and living conditions}

Approximately one in five older adults in the UK live in poverty. ${ }^{29}$ Several cross-sectional studies have demonstrated an association between lower financial income and greater frailty in later life $e^{25,26,31}$ as well a direct relationship between level of neighbourhood deprivation at birth and overall life expectancy and disability free life expectancy. ${ }^{29}$ This is to the extent that an individual born in the most affluent neighbourhood in the UK would, on average, expect to experience an additional 17 years of disability-free life, compared to the most deprived. ${ }^{32}$

Likewise, fewer years in education has been associated with greater frailty in older age, ${ }^{31}$ although this association attenuates when controlled for co-morbidities. ${ }^{33}$ A similar effect has been seen for employment, where greater frailty was observed among people who had performed typically lower paid and physically more challenging occupations. ${ }^{26,34}$ Additionally, evidence indicates that people in higher paid employment experience better mental health in early old age than those in lower paid employment. ${ }^{35}$

In addition to contributing to frailty development and progression, financial barriers may result in increased mortality for those with advancing frailty. A notable example is in reports on excess winter deaths. In the year 2012-13 an estimated 25,600 excess winter deaths occurred in England and Wales among those aged 75 and over. ${ }^{36}$ In addition to deaths from respiratory disease, due to an increased prevalence of influenza, these deaths were also due to cerebrovascular and ischaemic heart diseases. ${ }^{36,37}$ These are thought to be secondary to the effect of cold homes on increasing blood pressure, haemoconcentration and immunosuppression. These are key stressors for an older person living with frailty and impaired physiological reserve. ${ }^{38}$

\section{Role of the individual clinician}

> Identifying vulnerable patients and ensuring their awareness of available benefits as well as education regarding the importance of adequate home heating and implementing a Care Act Assessment. ${ }^{39}$

\section{Resilience}

Resilience has been defined as the process of effectively negotiating, adapting to or managing significant sources of stress or trauma. Assets and resources of the individual, their life and environment facilitate this capacity for adaptation and 'bouncing back' in the face of adversity. ${ }^{40}$ This definition of resilience aligns well with a framework around which policy responses to the wider determinants may be developed to build resilience at an individual and community level.

The large majority of resilience measures reflect the availability of assets and resources to facilitate resilience. They can therefore be useful for the identification of the presence or absence of these assets and resources, or as a method of measuring the process leading to a resilient outcome..$^{41}$ An example of a resilience scale is provided (Box 1$).^{42}$

There is a negative association between resilience and frailty. ${ }^{43}$ Factors that promote resilience, including physical health, a sense of self, social belonging, self-belief and altruism overlap with factors that reduce frailty. ${ }^{26,44-46} \mathrm{~A}$ useful way to conceptualise the relationship between frailty and resilience is that frailty can be considered to represent vulnerability to major changes in health following minor stressor events, whereas resilience represents the capability of an individual to adapt and bounce back following a stressor.

\section{Role of the individual clinician}

$>$ Awareness of resilience and its utilisation in identifying individuals at increased risk of frailty and greater vulnerability to stressor events.

Frailty, resilience and the wider determinants of health: coordinating the response

Building the resilience of older people living with frailty requires a coordinated response across central government, local authorities, the NHS, voluntary sector, individuals, families and communities. ${ }^{29}$ 


\section{Box 1. The Brief Resilience Scale}

1. I tend to bounce back quickly after hard times

2. I have a hard time making it through stressful events

3. It does not take me long to recover from a stressful event

4. It is hard for me to snap back when something bad happens

5. I usually come through difficult times with little trouble

6. I tend to take a long time to get over set-backs in my life

The Brief Resilience Scale is scored by reverse coding items 2, 4, and 6 and finding the mean of the six items. The following instructions are used to administer the scale: 'Please indicate the extent to which you agree with each of the following statements by using the following scale: $1=$ strongly disagree 2 = disagree, 3 =neutral, 4 = agree, 5 = strongly agree.' Reprinted with permission from Smith BW, Dalen J, Wiggins K et al. The Brief Resilience Scale: assessing the ability to bounce back. Int J Behav Med 2008;15:196.

This is highly complex, requiring engagement across a broad range of central government departments, for example the Department of Health and Social Care, the Department of Work and Pensions and the Ministry of Housing, Communities \& Local Government to ensure coordinated central policy. National specialist societies such as the British Geriatrics Society, voluntary sector organisations and royal colleges have a key potential role in central government engagement, and targeting the social determinants of health is a key component of the RCP strategy 2015-2020.47 The response at local level should be based around community engagement and empowerment, commissioning of whole-system, integrated, evidence-based services and shifting resources to local communities. Local clinicians have a key role to play in this context to ensure development, commissioning and provision of appropriately tailored services.

National variation in deprivation levels and contributory factors from the wider determinants mean different approaches will be required in different localities. This provides a useful means of engaging local communities to ensure that interventions are relevant and fit for purpose, enhancing participation and developing a sense of community. This can, in turn, culture a positive physical and social community, building overall community or social capital. The creation of social capital not only enhances the resilience of those involved by creating a social buffer against individual strain but also ensures that social interventions which are developed are sustained, thus enhancing their success. ${ }^{29}$ Examples of such programmes are expert patients programs, community health champions, housing and community improvement schemes, the creation of green spaces, and recreational and cultural facilities. ${ }^{48,49}$ The development of social capital and community involvement is particularly important where barriers to engagement exist such as poor mobility which is common in frailty.

A notable step forward has been the inclusion of routine frailty identification and management as a key component of the 2017-18 general medical services (GMS) contract, which is the contractual arrangement between general practitioners and the NHS in England. ${ }^{50}$ The GMS contract implementation has been supported through the national availability of the electronic frailty index (eFI), ${ }^{2}$ which is based on the cumulative deficit model and uses routinely available primary care electronic health record data to assist in the identification of frailty, supported by subsequent clinical confirmation. ${ }^{50}$ The national availability of the eFI and GMS contract developments may provide the framework for local development and commissioning of integrated, whole-systems solutions to build resilience for older people with varying degrees of frailty, based around personal and community assets and resources. Additionally, better knowledge of frailty prevalence at national and local level means that resources can be targeted towards communities with higher levels of coexisting frailty and deprivation, who are likely to be at especially high risk of adverse outcomes. A further advantage is the identification of a more standardised population for the development, testing and evaluation of novel interventions and services.

A promising potential approach to achieve this necessary shift is through personalised care planning, which is an anticipatory, negotiated series of discussions to clarify goals, options and preferences, and develop an agreed plan of action. ${ }^{51}$ Shared decision making is a crucial mechanism, providing linkage to additional mechanisms for improving outcomes through more effective self-management, better care coordination, and better access to community resources. In the UK, the Personalised Care Planning to Improve Quality of Life for Older People with Frailty (PROSPER) research programme is testing this new approach, in partnership with Age UK. ${ }^{52}$

\section{Conclusion}

Evidence indicates that the wider determinants of health accelerate the development and progression of frailty, driving health inequalities in later life. The associations between lower socioeconomic position, frailty and disability in older age are especially notable because of the potential impact on future population care needs in later life. Historically, research studies have predominantly focused on biological mechanisms of frailty and health service-orientated treatments, potentially underestimating the role of the wider determinants. Targeting the wider determinants provides a useful framework for building resilience in frailty from a personal and community perspective. This requires a coordinated response across central government, local authorities, the NHS, voluntary sector, individuals, families and communities, focused on developing, testing and commissioning evidence-based services for older people living with frailty.

\section{References}

1 British Geriatrics Society. Fit for Frailty - consensus best practice guidance for the care of older people living in community and outpatient settings. London: BGS, 2014.

2 Clegg A, Young J, Iliffe S et al. Frailty in elderly people. Lancet 2013:381:752-62.

3 NHS England. Older people living with frailty. NHS England. www.england.nhs.uk/ourwork/Itc-op-eolc/older-people/frailty [Accessed 26 July 2018].

4 Fried LP, Tangen CM, Walston ] et al. Frailty in older adults: evidence for a phenotype. J Gerontol A Biol Sci Med Sci 2001;56:M146-56.

5 Rockwood K, Song X, MacKnight C et al. A global clinical measure of fitness and frailty in elderly people. CMAJ 2005;173:489-95.

6 Morley JE, Vellas B, van Kan GA et al. Frailty consensus: a call to action. J Am Med Dir Assoc 2013:14:392-7.

7 Rodriguez-Mañas L, Fried LP. Frailty in the clinical scenario. Lancet 2015;385:e7-9.

8 Clegg A, Young J. The frailty syndrome. Clin Med 2011;11:72-5.

9 Beswick AD, Rees K, Dieppe P et al. Complex interventions to improve physical function and maintain independent living in elderly people: a systematic review and meta-analysis. Lancet 2008;371:725-35. 
10 Ellis G, Whitehead MA, Robinson D et al. Comprehensive geriatric assessment for older adults admitted to hospital: meta-analysis of randomised controlled trials. BMJ 2011;343:d6553.

11 Clegg A, Hassan-Smith Z. Frailty and the endocrine system. Lancet Diabetes Endocrinol 2018;6:743-52.

12 World Health Organization. Health Impact Assessment (HIA): The determinants of health. Geneva: WHO. www.who.int/hia/evidence/ doh/en [Accessed 26 July 2018].

13 World Health Organization. Social determinants of health. Geneva: WHO. www.who.int/social_determinants/sdh_definition/en [Accessed 21 September 2018].

14 Demakakos P, Biddulph JP, de Oliveira C et al. Subjective social status and mortality: the English longitudinal study of ageing. Eur ] Epidemiol 2018;33:729-39.

15 Matthews RJ, Smith LK, Hancock RM et al. Socioeconomic factors associated with the onset of disability in older age: a longitudinal study of people aged 75 years and over. Soc Sci Med 2005;61:1567-75.

16 Lang IA, Hubbard RE, Andrew MK et al. Neighborhood deprivation, individual socioeconomic status, and frailty in older adults. J Am Geriatr Soc 2009;57:1776-80.

17 Fusion48. FrailtyToolkit. www.frailtytoolkit.org/frailty-overview [Accessed 21 September 2018].

18 Whitehead M, Dahlgren G. What can be done about inequalities in health? Lancet 1991:338:1059-63.

19 Dahlgren G, Whitehead M. Policies and strategies to promote social equity in health. Stockholm: Institute for Futures Studies, 1991:11.

20 Kojima G, Iliffe S, Walters K. Smoking as a predictor of frailty: a systematic review. BMC Geriatr 2015;15:131.

21 Theou O, Blodgett JM, Godin J et al. Association between sedentary time and mortality across levels of frailty. CMAJ 2017;189:E1056-64.

22 Blodgett J, Theou O, Kirkland S et al. The association between sedentary behaviour, moderate-vigorous physical activity and frailty in NHANES cohorts. Maturitas 2015;80:187-91.

23 Rietman ML, van der AD, van Oostrom SH et al. The association between BMI and different frailty domains: A U-shaped curve? J Nutr Health Aging 2018;22:8-15.

24 Clegg AP, Barber SE, Young JB et al. Do home-based exercise interventions improve outcomes for frail older people? Findings from a systematic review. Rev Clin Gerontol 2011;22:68-78.

25 Thompson MQ, Theou O, Yu S et al. Frailty prevalence and factors associated with the frailty phenotype and frailty index: Findings from the North West Adelaide health study. Australas ] Ageing 2018;37:120-6.

26 Woo J, Goggins W, Sham A et al. Social determinants of frailty. Gerontology 2005;51:402-8.

27 Davidson S, Rossall P. Evidence review: Loneliness in later life. London: Age UK, 2015.

28 Oxley ], Corben B, Fildes B, O'Hare M, Rothengatter T. Older vulnerable road users - measures to reduce crash and injury risk. Victoria: Monash University Accident Research Centre, 2004.

29 Strategic Review of Health Inequalities in England post-2010. Fair society, healthy lives: The Marmot review. London: The Marmot Review, 2010.

30 Lloyd-Sherlock P, Agrawal S. Fear of crime and its effects on older people: Comparing six countries. London: Age UK, 2014

31 Romero-Ortuno R. Frailty index in Europeans: association with determinants of health. Geriatr Gerontol Int 2014;14:420-9.

32 Office For National Statistics. Health state life expectancies by national deprivation deciles, England and Wales: 2014 to 2016 ONS, 2018

33 Syddall H, Roberts HC, Evandrou M et al. Prevalence and correlates of frailty among community-dwelling older men and women: findings from the Hertfordshire cohort study. Age Ageing 2010;39:197-203.
34 Iavicoli I, Leso V, Cesari M. The contribution of occupational factors on frailty. Arch Gerontol Geriatr 2018;75:51-8.

35 Chandola T, Ferrie J, Sacker A et al. Social inequalities in self reported health in early old age: follow-up of prospective cohort study. BMJ 2007:334:990.

36 Office for National Statistics. Excess winter mortality in England and Wales: 2011-12 (provisional) and 2010-11 (final). Newport: ONS, 2012.

37 The Eurowinter Group. Cold exposure and winter mortality from ischaemic heart disease, cerebrovascular disease, respiratory disease, and all causes in warm and cold regions of Europe. Lancet 1997:349:1341-6

38 Woodhouse PR, Khaw KT. Plummer M. Seasonal variation of blood pressure and its relationship to ambient temperature in an elderly population. J Hypertens 1993;11:1267-74.

39 Department of Health \& Social Care. Guidance: Care Act factsheets. London: Department of Health \& Social Care, 2016. www.gov.uk/government/publications/care-act-2014-part-1factsheets/care-act-factsheets [Accessed 18 ]anuary 2019].

40 Windle $\mathrm{G}$. What is resilience? A review and concept analysis. Rev Clin Gerontol 2010;21:152-69.

41 Windle G, Bennett KM, Noyes ]. A methodological review of resilience measurement scales. Health Qual Life Outcomes 2011;9:8.

42 Smith BW, Dalen J, Wiggins K et al. The brief resilience scale: assessing the ability to bounce back. Int J Behav Med 2008;15:194-200.

43 Freitag S, Schmidt S. Psychosocial Correlates of Frailty in Older Adults. Geriatrics 2016;1:26.

44 Levine S. Psychological and social aspects of resilience: a synthesis of risks and resources. Dialogues Clin Neurosci 2003;5:273-80.

45 Whitson HE, Duan-Porter W, Schmader KE et al. Physical resilience in older adults: Systematic review and development of an emerging construct. J Gerontol A Biol Sci Med Sci 2016;71:489-95.

46 Rebagliati GA, Sciume L, Iannello $P$ et al. Frailty and resilience in an older population. The role of resilience during rehabilitation after orthopedic surgery in geriatric patients with multiple comorbidities. Funct Neurol 2016;31:171-7.

47 Royal College of Physicians. RCP strategy 2015-2020. London: RCP, 2015.

48 National Primary Care Research and Development Centre. The national evaluation of the pilot phase of the Expert Patient Programme: Final report. NPCRDC, 2006.

49 James K. Prescribing Learning: A Guide to Good Practice in Learning and Health. Leicester National Institute of Adult Continuing Education, 2001.

50 NHS England GP Contracts Team. NHS England standard general medical services contract 2017/18. London: NHS England, 2017.

51 Coulter A, Entwistle V, Eccles A et al. Personalised care planning for adults with chronic or long-term health conditions. Cochrane Database Syst Rev 2015:CD010523.

52 Clegg A. Personalised Care Planning to Improve Quality of Life for Older People with Frailty (PROSPER). National Institute for Health Research Programme Grant for Applied Research (RP-PG-0216200003).

Address for correspondence: Dr Matthew Hale, Academic Unit of Elderly Care and Rehabilitation, University of Leeds, Bradford Teaching Hospitals NHS Foundation Trust, Bradford BD9 6RJ, UK.

Email: m.hale@doctors.org.uk 